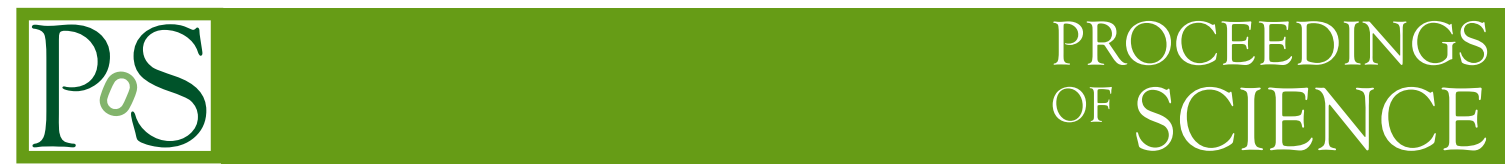

\title{
Optimization in Tensor Polarized Targets
}

\section{Dustin Keller*†}

University of Virginia

E-mail: dustindjlab.org

\begin{abstract}
A look into dynamic orientation using optimized radio-frequency (RF) irradiation produced perpendicular to the holding field is presented for the spin-1 system required for tensor polarized fixed target experiments. The application of RF from a coil around the target material produced close to the Larmor frequency of the nucleus can be used to study the manipulation of the quadrupole polarization. A framework to understand the frequency domain of the quadrupole and nuclear polarization is also discussed.
\end{abstract}

XVIth International Workshop in Polarized Sources, Targets, and Polarimetry 14-18 September 2015

Ruhr-University Bochum, Germany

\footnotetext{
*Speaker.

${ }^{\dagger}$ for UVA Polarized Target Group
} 


\section{Introduction}

A polarized target system cools $(T \leq 1 \mathrm{~K})$ a polarizable material while in a homogeneous high magnetic field. Photon scattering experiments typically use a ${ }^{3} \mathrm{He} /{ }^{4} \mathrm{He}$ dilution refrigerator so that the desired target ensemble helicity can be held in a frozen spin state. For frozen spin targets large negative quadrupole enhancement is achievable in a transient state which can be rendered useful as a target by the reduction of the relaxation rates at the low temperatures $(T \leq 100 \mathrm{mK})$ in the frozen spin mode. This of course is less practical for charged beam experiments where interfacial thermal resistance of the solid target limits the beam current. For electron or proton beam scattering experiments a ${ }^{4} \mathrm{He}$ evaporation refrigerator is required in combination with a powerful pumping system. Manipulation of the spin-1 magnetic sublevels using RF is different for frozen spin and evaporation temperature targets. Here the focus is on the latter.

High polarization of solid nuclear targets is achieved with dynamic nuclear polarization (DNP) using a material that is radiation resistant and doped with a system of paramagnetic centers $(2 \times$ $10^{19}$ spins $/ \mathrm{cm}^{3}$ at $5 \mathrm{~T}$ ). The alignment of a spin- 1 target naturally increases through the DNP process when the magnetic sublevels are filled following a Boltzmann distribution. However maximizing the alignment outside of equilibrium is of great interest for the purpose of quadrupole (tensor) polarization enhancement. The figure of merit (FOM) for a solid tensor polarized target can be defined as,

$$
F O M=n_{t} f^{2} Q_{n}^{2}
$$

where $n_{t}$ is the target thickness, $f$ is the ratio of the number of polarizable nucleons to the total number of nucleons in the target material, and $Q_{n}$ is the degree of quadrupole polarization. The magnitude of the quadrupole polarization also sets the scale of the asymmetries that are accessible. Normalization factors that can change with time in each target helicity state lead to systematic errors or drifts in asymmetry. The false asymmetries produced from these drifts are expressed as,

$$
\delta A_{z z}=\frac{2}{f P_{z z}} \delta \zeta
$$

where $\delta \zeta$ is the combination of effects from accumulated charge, detector efficiency, and all possible contributions to the drift in the observable.

The instrumental aspects of systematic uncertainty $(\delta \zeta)$ that govern the scale of the observable that can be investigated with this type of experiment are the true limiting factors. Polarization enhancement can help to suppress these effects but it can only be one element of a multipronged approach to address contribution to $\delta \zeta$ having nothing to do with polarization or polarization uncertainty. For a more detailed outline of the polarization systematic uncertainty and minimization procedure refer to [1]].

\section{Spin-1 Polarized Target in $1 \mathrm{~K}$}

Dynamic Nuclear Polarization (DNP) of the solid material used in nuclear experiments can be achieved at $\sim 1 \mathrm{~K}$ using a homogeneous magnetic holding field and a microwave field to transfer the spin polarization from the electrons to the nuclei. The solid polarized target system, see Figure 1 and Figure 日, uses a ${ }^{4} \mathrm{He}$ evaporation refrigerator with sufficiently high cooling power to minimize 


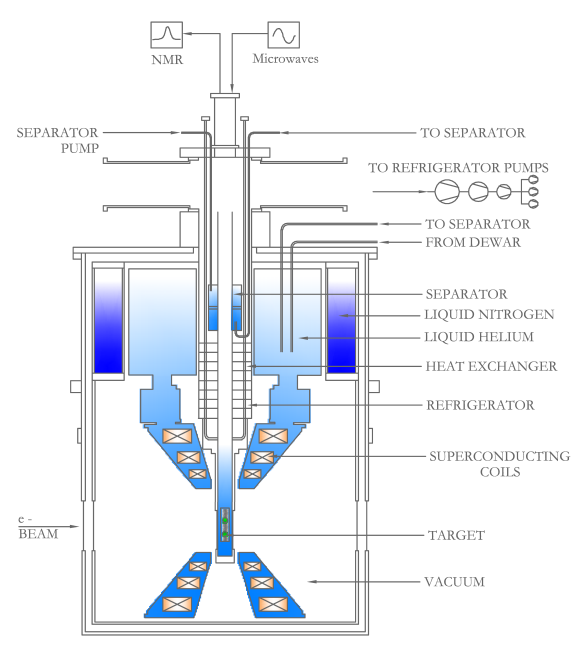

Figure 1: Polarized target system for $\mathrm{NH}_{3} / \mathrm{ND}_{3}$ at $5 \mathrm{~T}$ used to achieve a temperature of $\sim 1 \mathrm{~K}$.

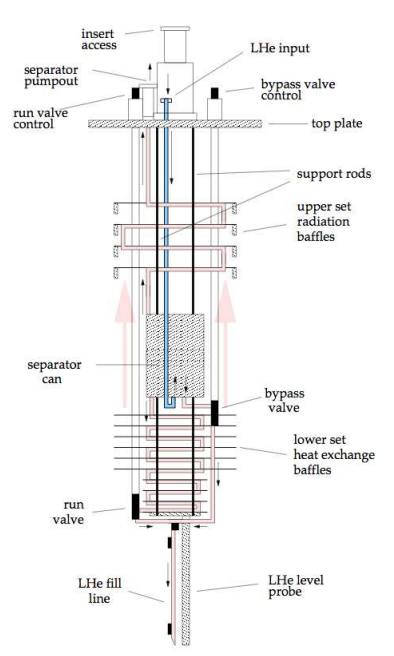

Figure 2: The fridge of the target system.

the heating effects of the microwaves and high beam current. A superconducting magnet is used to polarize and maintain polarization of the target material during the experiment. The nuclear spin polarization is measured with a continuous-wave NMR coil and Liverpool Q-meter [2]. The Q-meter works as part of a circuit with phase sensitivity designed to respond to the change of the impedance in the NMR coil. The radio frequent (RF) susceptibility of the material is inductively coupled to the NMR coil which is part of a series LCR circuit, tuned to the Larmor frequency of the nuclei being probed. The output, consisting of a DC level subtracted by a post-Q-meter conditioning card (Yale card), is digitized and recorded as a target event [3].

Measurements of the deuteron polarizations using CW-NMR contain more information for the polycrystalline materials such as deuterated butanol and deuterated ammonia. The two overlapping absorption lines are analyzed to determine the signal intensities $I_{+}, I_{-}$resulting in an approximated relative population of the magnetic sublevels. When these sublevels exist under a Boltzmann distribution obtaining polarizations are particularly simple and with well managed errors. The target spin orientation can be described using the vector and tensor polarization. Tensor polarization can be expressed in terms of the vector polarization as $P_{z z} \doteq 2-\sqrt{4-3 P^{2}}$ where the dot implies the relation is true when thermal equilibrium exists within the deuteron spin species. The definition of vector polarization for spin-1 is,

$$
P=\frac{n_{+}-n_{-}}{n_{+}+n_{-}+n_{0}} \doteq \frac{r^{2}-1}{r^{2}+r+1},
$$

with the tensor polarization defined as,

$$
P_{z z}=\frac{n_{+}-2 n_{0}+n_{-}}{n_{+}+n_{-}+n_{0}} \doteq \frac{r^{2}-2 r+1}{r^{2}+r+1} .
$$

Where the second equality in each case is not part of the definition but a relation to the transition ratio $r$ defined as $r=I_{+} / I_{-}$, see Figure 3. These relations in terms of $r=e^{\beta \hbar \omega_{d}}$ are true to first 


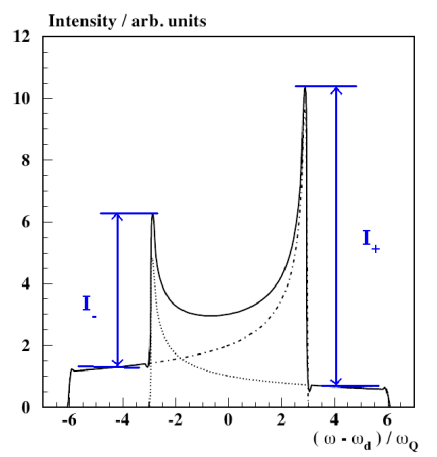

Figure 3: Deuteron Magnetic Resonance line shape and peak intensities $I_{+}$and $I_{-}$.

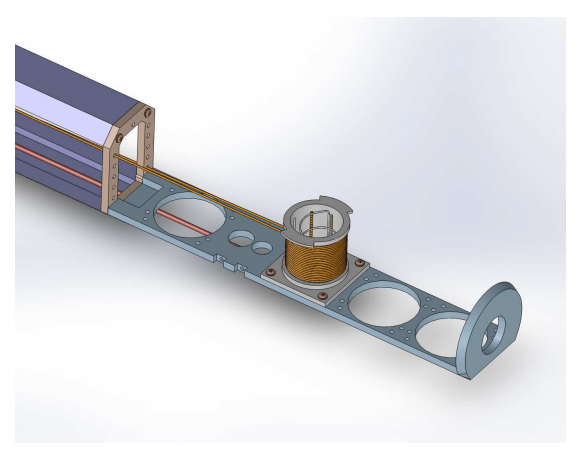

Figure 4: Target cup and coil used to generate the modulated RF field.

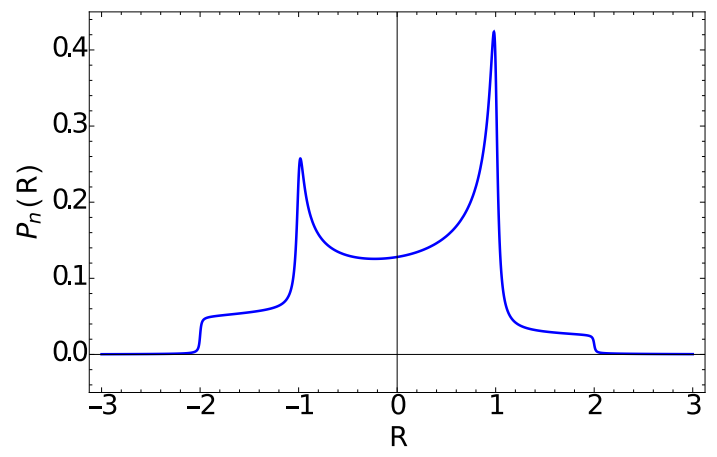

Figure 5: The nuclear polarization as a function of line position $P_{n}(R)$ for a nuclear polarization of $42 \%$.

order in $\beta \hbar \omega_{q}$, where $\omega_{d}\left(\omega_{q}\right)$ is Larmor (quadrupole interaction) frequency. The intensities $I_{-}$and $I_{+}$can be determined using a fitting procedure [4]. The resulting polarization measurements can be used complementary to the TE signal polarization calibration. For vector polarizations over $30 \%$ the two independent methods result in a discrepancy smaller than $2.5 \%$. This leads to a minimum uncertainty in the natural tensor polarization of approximately $5 \%$.

\section{Enhanced Tensor Polarization}

Deuteron spin alignment can be manipulated when exposed to a modulated RF field using an external coil around the target cup, as seen if Figure $\emptyset$. The rate equations for the target material can be used to understand the change of transition intensities at the irradiated NMR line positions. The results of the dynamic solutions of the overlapping absorption lines define the change in amplitude of the lineshape at each frequency. There are many polarization response characteristics of the RF that must be accounted for to determine the intensity at every frequency. Each absorption line as a function of time includes both longitudinal and transverse aspects of the polarization. To interpret the intensities at a particular frequency or line position $R=\left(\omega-\omega_{d}\right) / \omega_{q}$ it is convenient to use $I_{+}(R)$ and $I_{-}(R)$. One can then define the nuclear $P_{n}(R)$ and quadrupole $Q_{n}(R)$ 


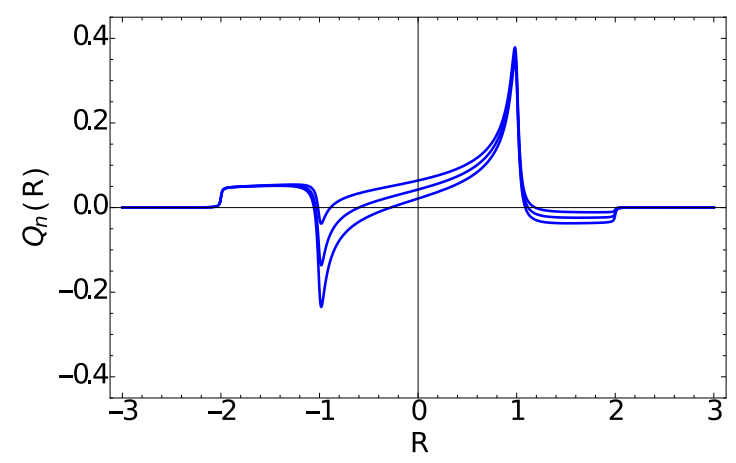

Figure 6: The quadrupole polarization as a function of line position $Q_{n}(R)$ for a nuclear polarization of $19 \%, 42 \%$, and $71 \%$. Optimization of the quadrupole polarization is obtained by negating the values that drop below zero using RF induced transition for that select frequency range.

polarization at a given line position $R$. The nuclear polarization over line position $R$ as define by $P_{n}(R)=C\left[I_{+}(R)+I_{-}(R)\right]$ is shown in Figure 5 . The lineshape is only relevant when the magnetic sublevels are in a Boltzmann distribution. Tensor target optimization for positive or negative nuclear polarization occurs when negative quadrupole polarization has been minimized through the frequency domain. The distribution of negative quadrupole polarization change about the $R=0$ axis for negative nuclear polarization. Analytic minimization is easily seen for the positive case by studying the quadrupole polarization as a function of frequency. An example where the quadrupole polarization as a function of line position $Q_{n}(R)$ as defined by $Q_{n}(R)=C\left[I_{+}(R)-I_{-}(R)\right]$ is shown in Figure 6 for a nuclear polarization of $19 \%, 42 \%$, and $71 \%$. Again, the lineshape is only relevant when the magnetic sublevels are in a Boltzmann distribution. To maximize the positive quadrupole polarization of the sample the magnitude of $Q_{n}(R)$ that drops below zero must be minimized with modulated RF across the required resonance frequencies. A new lineshape evolves taking into account the magnetization response characteristics of the modulated $\mathrm{RF}$ at each position $R$. The FOM of the scattering experiment is maximized when $P_{n}(R)$ is greatest for $Q_{n}(R)>0$ over the course of data taking.

\section{Conclusion}

Maximizing tensor target polarization for a charged beam spin- 1 target is done by using DNP on the target material in the standard way and then dynamically orienting the target using optimized radio-frequency (RF) irradiation produced perpendicular to the holding field to minimize the opposite signed quadrupole polarization over the frequency domain. The theoretical framework and data studies are being developed at the University of Virginia Polarized Target Group and will be published in the near future.

\section{References}

[1] D. Keller, Nucl. Instr. and Meth. A728:133 (2013) 
[2] G.R. Court, et al. Nucl. Instr. and Meth. A324:433 (1993)

[3] D. Crabb, W. Meyer Solid Polarized Targets for Nuclear and Particle Physics Experiments Nucl. Part. Sci. 47:67-109 (1997)

[4] C. Dulya, et al. Nucl. Instr. and Meth. in Phys. Res. A 398, 109-125(1997)

[5] G.R. Court, et al. Nucl. Instr. and Meth. in Phys. Res. A 527, 253 (2004)

[6] de Boer, W. et al. Phys.Lett. B46 143-144 (1973) 